\title{
VERIFICATION OF BEHAVIOUR OF HUMAN ENAMEL FOR FRACTURE TOUGHNESS DETERMINATION
}

\author{
Petra HÁJková*, Aleš Jíra, Luboš Řehounek \\ Czech Technical University in Prague, Faculty of Civil Engineering, Thákurova 7166 29, Prague, Czech \\ Republic \\ * corresponding author: petra.hajkova@fsv.cvut.cz
}

\begin{abstract}
Enamel is the hardest biological tissue in the human body because of its structure and composition. The structure of interlocking rods enables this biomaterial to resist the stresses of mastication. Unfortunately, enamel is prone to fracture initiation and growth. Determining the fracture toughness of enamel is a difficult task. The lack of thickness makes it impossible to prepare samples which could be analysed by usual methods. Other authors ordinarily use Vickers indentation fracture test (VIF) to determine the fracture toughness of enamel. The VIF is, however, not generally acceptable.

The aim of this study is a verification of a fracture behaviour of enamel using nanoindentation. In the study the impact of changes of hardness $\left(H_{\mathrm{IT}}\right)$ and reduced modulus $\left(E_{\mathrm{r}}\right)$ caused by crack initiation and the growth on fracture toughness determination is observed. The next goal is an evaluation of loading rate impact.
\end{abstract}

KEYworDs: human enamel, fracture behaviour, mechanical properties, loading rate, work of indentation.

\section{INTRODUCTION}

Enamel is a tissue that forms the external part of human teeth. Conventionally, it is the only visible dental material in the oral cavity. Enamel also protects the inner parts of the human teeth - dentin and pulp. Its mechanical properties correspond to its functions and conditions it is under - enamel must withstand the stresses caused by mastication and other stimuli, such as sudden changes of temperature caused by drinking hot or cold drinks and consuming acidic or sugary foods. Because of that, enamel is the hardest tissue in the human body [1]. Unfortunately, hard materials also tend to be brittle and exhibit little resistance towards initiation and propagation of cracks. The cracks can then compromise the overall health of the tooth, especially if dental cavities are located beneath the enamel layer.

Mechanical properties of enamel are determined by its structure and chemical composition. It consists of $96 \%$ anorganic substances (hydroxyapatite) and $4 \%$ is water and organic compounds (protein enamelin). Densely formed crystals of hydroxyapatite form prisms that pass in volume from the dentino-enamel junction (DEJ) to the outer tooth layer. The shape of the prisms enables for the interconnection of convex and concave surfaces of surrounding prisms and forms a strong bond. The different orientation of the hydroxyapatite crystals in prisms and the interprismatic substance also contributes towards a higher mechanical durability [1].

Authors who describe the mechanical properties of dental tissues have previously dedicated more at- tention to dentin than enamel. This is caused by the fact that samples of healthy, intact enamel are hard to come by and it has a maximum thickness of $2.5 \mathrm{~mm}$. The thickness of the samples also limits the number of viable testing methods. The most common method used for the determination of hardness $H_{\text {IT }}$ and Young's Modulus $E$ of dentin and enamel is nanoindentation. Enamel, which is a very brittle material, has another important property - the fracture toughness.

Fracture toughness describes the resistance of the material against the propagation of cracks at given stress values. The property most commonly used for describing the fracture toughness of enamel is the stress intensity factor $K_{\text {IC }}$. The mean value of the fracture toughness of enamel, according to a published research, ranges between $0.45-1.55 \mathrm{MPa} \mathrm{m}^{1 / 2}$ [2]5]. A great range of experimentally determined values is caused by multiple factors. Authors [2, 3] mention an increasing brittleness from the DEJ towards the outer tooth surface for old enamel $(50 / 55 \leq$ age). Other published works mention the different properties of enamel in deciduous and permanent teeth [4] and differences caused by the enamel structure - indentation parallel or perpendicular to enamel rods [5]. The problems connected with the determining the values of the fracture toughness of enamel do not stem only from the inhomogeneous nature of enamel and its time-dependent changes but also from the chosen testing method. Although authors almost solely use the microindentation and Vickers indentation fracture test (VIF) for fracture toughness determination of 
enamel, the formulas used for calculating $K_{\mathrm{IC}}$ differ. The most common formula used to calculate values of $K_{\mathrm{IC}}$ is as follows 6 :

$$
K_{\mathrm{IC}}=\delta\left(\frac{E}{H_{\mathrm{IT}}}\right)^{1 / 2} \frac{P}{a^{3 / 2}}
$$

where $E$ is the Young's Modulus, $H_{\mathrm{IT}}$ is the hardness, $P$ is the applied load, a is the crack length and $\delta$ is calibration constant. The differences in the values of calibration constants seem to be one of the main reasons for the different outcomes among various researchers. Authors also use other formulas, which take into account other parameters, such as the size of the indent, the face angle of the indentation tip etc. Because of these discrepancies, the VIF method is not seen as acceptable for the fracture toughness testing by some researchers. Quinn and Bradt, in their review [7, point out other problems of the VIF method. The problems are, for example, that the VIF method does not meet the definition of linear fracture mechanics as there is no crack in the specimen at the beginning of a test. The overall results can also be influenced by the polishing of a specimen, the range of applied stress, which causes initiation of different types of cracks (Palmqvist, median etc.), or accuracy of measurement (length of cracks). A part of the review was also dedicated to a comparison of results of fracture toughness, determined by the VIF method using three different popular equations, for standard reference material (SRM). As the authors expected, none of these equations yielded correct certified values of fracture toughness for the SRM.

The main goal of this study is to assess the brittle behaviour of enamel during nanoindentation tests, where the sample is loaded by $10-100 \times$ smaller forces than during the (micro)indentation, which is used in experiments for the fracture toughness determination of other authors [2 4, 8]. The possibility of using nanoindentation for fracture toughness determination by the VIF method is examined. Further effort is dedicated towards identifying the contribution of other factors to the overall results of the fracture toughness test. The work focuses on the variations of reduced modulus $E_{\mathrm{r}}$ and hardness $H_{\mathrm{IT}}$ as a result of initiation and propagation of cracks that form under $10-150 \mathrm{mN}$ force loads. Another observed factor that can influence the measurements is the loading velocity. At the end of this work an alternative method for the fracture toughness determination, which is based on a dissipation of energy, is presented.

\section{Materials And Methods}

\subsection{SPeCimen Preparation}

We chose a human molar with no visible defects as a test specimen for our research. The molar was cleaned of all impurities and embedded into a technical Dentacryl solution from the SpofaDental company. It was subsequently transversally cut from both sides to form a $15 \mathrm{~mm}$ wide specimen. The sections were made using a water-cooled saw cutter (ATM Brillant 210, Austria) with a diamond disc. The first section was situated above the tooth crown and the second inside its root. The sample was grinded with a coarse silicon-carbide (SiC) paper (coarseness 320 ) from the side of the crown until the section reached a desirable depth of enamel. Then it was grinded again with finer papers (coarseness 1000, 2500) and polished with a diamond paste $(0.25 \mu \mathrm{m})$. The thorough grinding and polishing was the most important part of the specimen preparation, because scratches in the surface of the specimens could impair the whole measurement. Finally, it was cleaned with ultrasonic waves.

\subsection{NANOINDENTATION Method}

Nanoindentation was performed on the CSM Instruments Nano Hardness Tester (Anton Paar, Austria) equipped with a diamond cube corner tip. The indentation tip was chosen with respect to the experiment as the cube corner tip has a smaller face angle $\left(35.26^{\circ}\right)$ compared to the Berkovich $\left(65.27^{\circ}\right)$ or Vickers $\left(68^{\circ}\right)$ tip, so it is sharper and induces the cracks easier. The sample was loaded gradually with the force increasing without oscillations. The unloading curve slope was the same as loading curve, using the same velocity. The force-controlled test was used for each cycle of indentation.

For determining the hardness $H_{\mathrm{IT}}$ and reduced modulus $E_{\mathrm{r}}$, the methodology of Oliver \& Pharr was used [9]. This method uses the unloading curve and the reduced modulus is calculated as follows:

$$
E_{\mathrm{r}}=\frac{\sqrt{\pi}}{2} \frac{S}{\sqrt{A}}
$$

where $S$ is the contact stiffness $(\mathrm{d} P / \mathrm{d} h)$ and $A$ is the contact area of the indent determined from the measured contact depth $h_{\mathrm{c}}$. Unlike Young's Modulus, the reduced modulus takes into account the fact that the measured contact depth is the sum of the specimen deformation and the deformation of the indentation tip itself.

Hardness, which represents the material's resistance to a localised plastic deformation induced by a mechanical indentation, is calculated from the maximum load $P_{\max }$ and contact area $A$ :

$$
H_{\mathrm{IT}}=\frac{P_{\max }}{A}
$$

For determining the value changes of $E_{\mathrm{r}}$ and $H_{\mathrm{IT}}$ due to initiation and propagation of cracks and evaluating the use of nanoindentation for measuring the fracture toughness of enamel $5 \times 16$ indents were performed. Each of these 5 matrices had a different value of maximum applied load (10, 20, 40, 80 and $150 \mathrm{mN}$ ). The indentation cycle of all matrices was identical. The loading consisted of 3 steps - the loading curve $(120 \mathrm{mN} / \mathrm{min})$, constant load (10 sec) and unloading $(120 \mathrm{mN} / \mathrm{min})$. The 10 -second long constant load was 

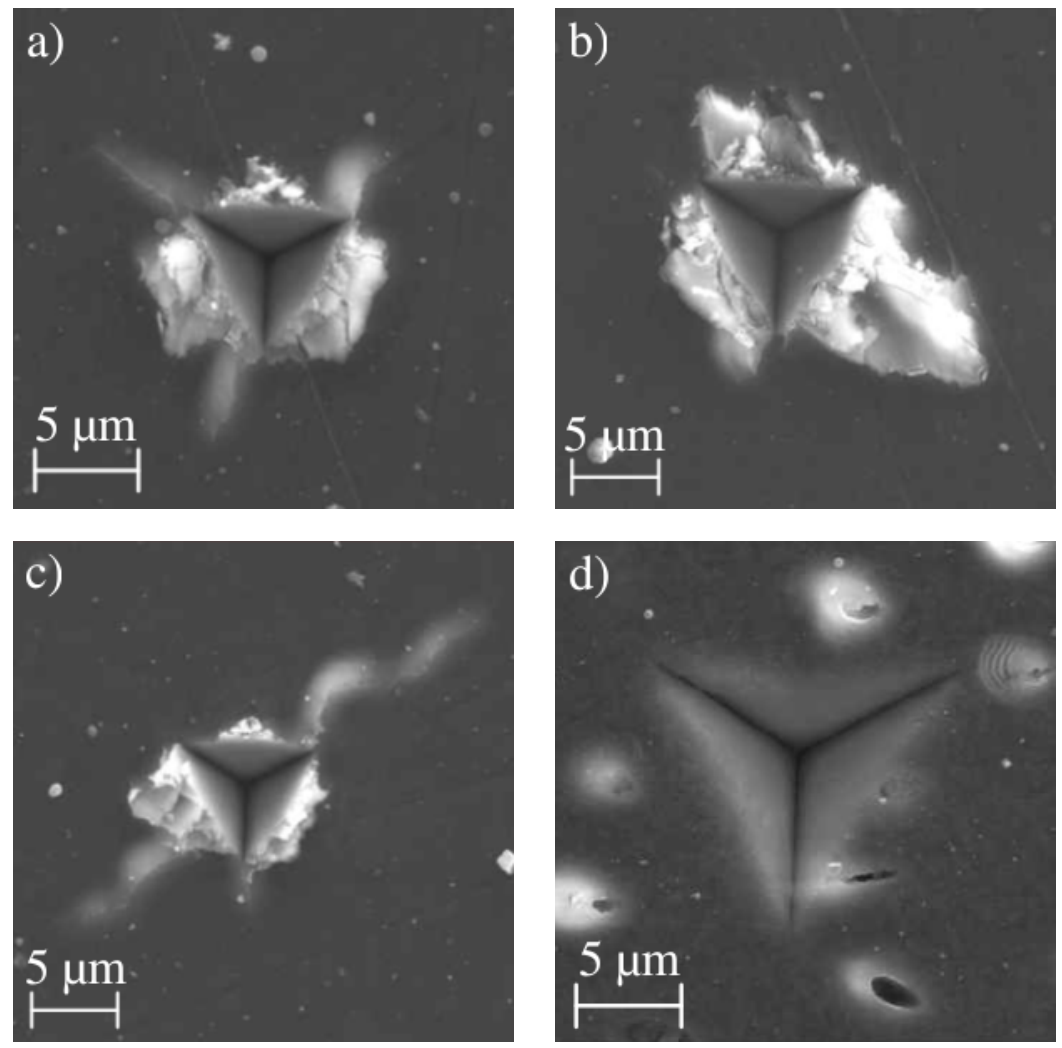

FiguRE 1. Picture of indents performed by scanning electron microscopy (Tescan MIRA, Czech Republic). Different types of cracks: a) Palmqvist or half-penny cracks, b) splitting off, delamination and c) radial cracks going through the indent. d) indent performed in dentin.

applied in order to eliminate the creep influence on the final results. The contact depth varied between 787-3759 $\mathrm{nm}$ depending on the maximum applied load.

The distance of individual indents was chosen in regard to the anticipated contact depth so the distance between the individual indents was at least $2 \times$ greater than the indent itself but also as small as possible so it would not be influenced by the inhomogeneities caused by the transition from the DEJ to the outer tooth surface. The distance, which is too small, can influence the final results due to an overlap of plastic areas.

For evaluating the influence of the load velocity on the brittle behaviour of enamel, $3 \times 25$ indents were performed. The maximum applied load $(150 \mathrm{mN})$ was identical for all indents. The maximum value of loading force applicable by the testing instrument was chosen in regard to the maximization of the crack propagation. The indentation cycle for individual matrices consisted of loading with the velocity of 60 , 250 and $450 \mathrm{mN} / \mathrm{min}$ up to the maximum value of force $(150 \mathrm{mN})$ and immediate unloading at the same velocity. The contact depth varied between 3260$3835 \mathrm{~nm}$. The distance between individual indents was $50 \mu \mathrm{m}$, just as during the previous experiment for the maximum value of $150 \mathrm{mN}$.

\section{Results}

The brittle behaviour of enamel during the nanoindentation, when the sample was loaded with forces of $10-150 \mathrm{mN}$, was different from the behaviour other authors describe for (micro)indentation and loading with forces of 1-10N [2, 4, 10]. They describe the initiation of either Palmqvist or half-penny cracks, which are necessary for the determination of fracture toughness by the VIF method. These cracks were rarely encountered in our study (Fig. 1 1 ) , splitting off and delamination (Fig. 1p) were seen more often. Radial cracks were also observed, but it did not radiate from all peaks of the indent, there was just one crack going through the indent (Fig. 1r). To highlight the enamel's brittleness and to show a comparison between different dental tissues, a picture describing the indent performed with the same force located in dentin (Fig. 1 d ) was also included. No cracks were visible in dentin that indicated greater fracture toughness of dentin than that of enamel. Determination of the mechanical properties of dentin was a part of our previous efforts [11, 12. The brittle behaviour of enamel has shown to be very heterogeneous and very difficult to be precisely defined.

The final values of fracture toughness can be influenced by many factors. Equation (1) shows that the fracture toughness determined by the VIF method depends on hardness $H_{\mathrm{IT}}$ and reduced modulus $E_{\mathrm{r}}$. Therefore, changes of these characteristics have to be 


\begin{tabular}{ccccc}
\hline Indentation Load $[\mathrm{mN}]$ & Loading Rate $[\mathrm{mN} / \mathrm{min}]$ & $h_{\mathrm{c}}[\mathrm{nm}]$ & $H_{\mathrm{IT}}[\mathrm{GPa}]$ & $E_{\mathrm{r}}[\mathrm{GPa}]$ \\
\hline 10 & 120 & $787 \pm 40$ & $3.79 \pm 0.402$ & $79.65 \pm 4.924$ \\
20 & 120 & $1139 \pm 65$ & $3.71 \pm 0.437$ & $80.33 \pm 4.618$ \\
40 & 120 & $1673 \pm 104$ & $3.50 \pm 0.386$ & $80.95 \pm 4.198$ \\
80 & 120 & $2491 \pm 114$ & $3.28 \pm 0.297$ & $81.55 \pm 5.276$ \\
150 & 120 & $3497 \pm 263$ & $3.25 \pm 0.421$ & $85.66 \pm 5.606$ \\
\hline 150 & 60 & $3470 \pm 239$ & $3.29 \pm 0.391$ & $85.33 \pm 6.456$ \\
150 & 250 & $3465 \pm 309$ & $3.31 \pm 0.500$ & $86.16 \pm 9.811$ \\
150 & 450 & $3534 \pm 300$ & $3.20 \pm 0.461$ & $82.22 \pm 9.070$ \\
\hline
\end{tabular}

TABLE 1 . Values of hardness $H_{\mathrm{IT}}$, reduced modulus $E_{\mathrm{r}}$ and contact depth $h_{\mathrm{c}}$ in relation to a) applied load and b) load velocity.
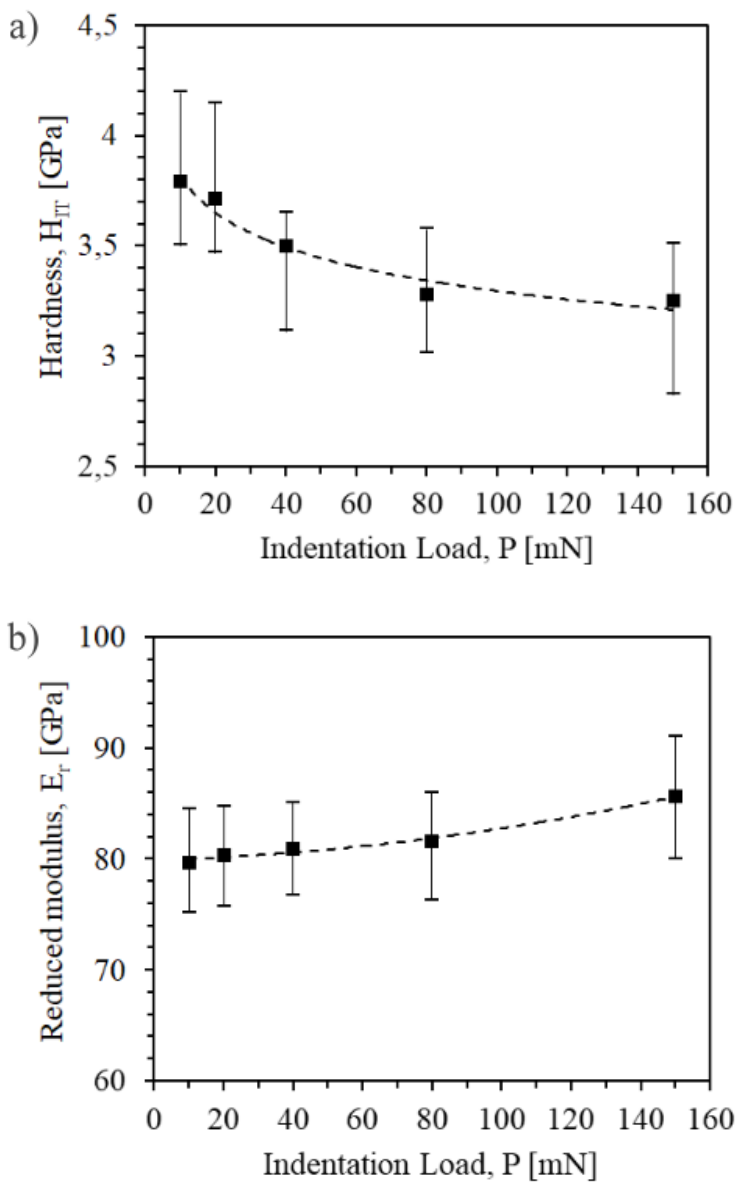

FIGURE 2. Graphs describing the changes of a) hardness $H_{\mathrm{IT}}$ and b) reduced modulus $E_{\mathrm{r}}$ due to changes of applied load.

one of the main factors affecting the output. Table 1 shows that different applied load influences the values of $H_{\mathrm{IT}}$ and $E_{\mathrm{r}}$. Values of hardness decreased with increasing load from $3.79 \mathrm{GPa}$ at $10 \mathrm{mN}$ to $3.25 \mathrm{GPa}$ at $150 \mathrm{mN}$ (Fig. 2a). The hardness decreased by $14 \%$ overall. The reduced modulus exhibited a different trend and increased with increasing load from 79.65 GPa to 85.66 GPa (Fig. 2p). The reduced modulus increased by $7 \%$ overall.

These changes are likely attributed to the initiation and propagation of cracks. The characteristic inden- tation curve (Fig. 3) obtained at the maximum load of $150 \mathrm{mN}$ shows the initiation of cracks and clarifies the changes in the values of micromechanical properties. Initiation and propagation of cracks cause an energy dissipation, which affects the indentation curve by a pop-in effect. Cracks formed in a close proximity of the indentation tip cause a sudden increase of contact depth $\Delta h_{\mathrm{c}}$. Methodology described in $\S 2.2$ implies that changes in contact depth influence the values of the contact area $A$ and, therefore, also the hardness (3) and reduced modulus (2). With regard to the fact that the contact area $A$ is the denominator in (2), we assumed a decreasing trend of the reduced modulus like in the case of hardness. Therefore, the opposite trend of the reduced modulus had to be the consequence of the increasing contact stiffness $S$.

It is possible to deduce that the values of hardness and reduced modulus measured at minimal applied load of $10 \mathrm{mN}$ are the most accurate. The indentation curves at this load did not show initiation and propagation of cracks. Verification of values is possible by applying lower loads, but the anticipated variation is negligible with regard to uncertainties of the fracture toughness calculation.

Table 1 also shows that the velocity of loading does not have any dramatic effect on the values of hardness, reduced modulus and crack initiation. Although the velocity of $450 \mathrm{mN} / \mathrm{min}$ caused a decrease of hardness by $2.7 \%$ (compared to the velocity of $60 \mathrm{mN} / \mathrm{min}$ ), no continuous decreasing trend was observed. The changes of the reduced modulus does not correspond to the previous experiment. In contrast to the increasing trend, in this experiment, the reduced modulus decreased by $3.8 \%$. It is possible that the decrease was caused by the location of the matrix of indents in enamel, which is inhomogeneous.

\section{Discussion}

The brittle behaviour of enamel during the nanoindentation test was found to be very heterogeneous. A number of different types of cracks were induced (Palmqvist, half-penny) but splitting off and delamination were prevalent. The lack of clear cracks emanating from corners of the indentation tip makes it 


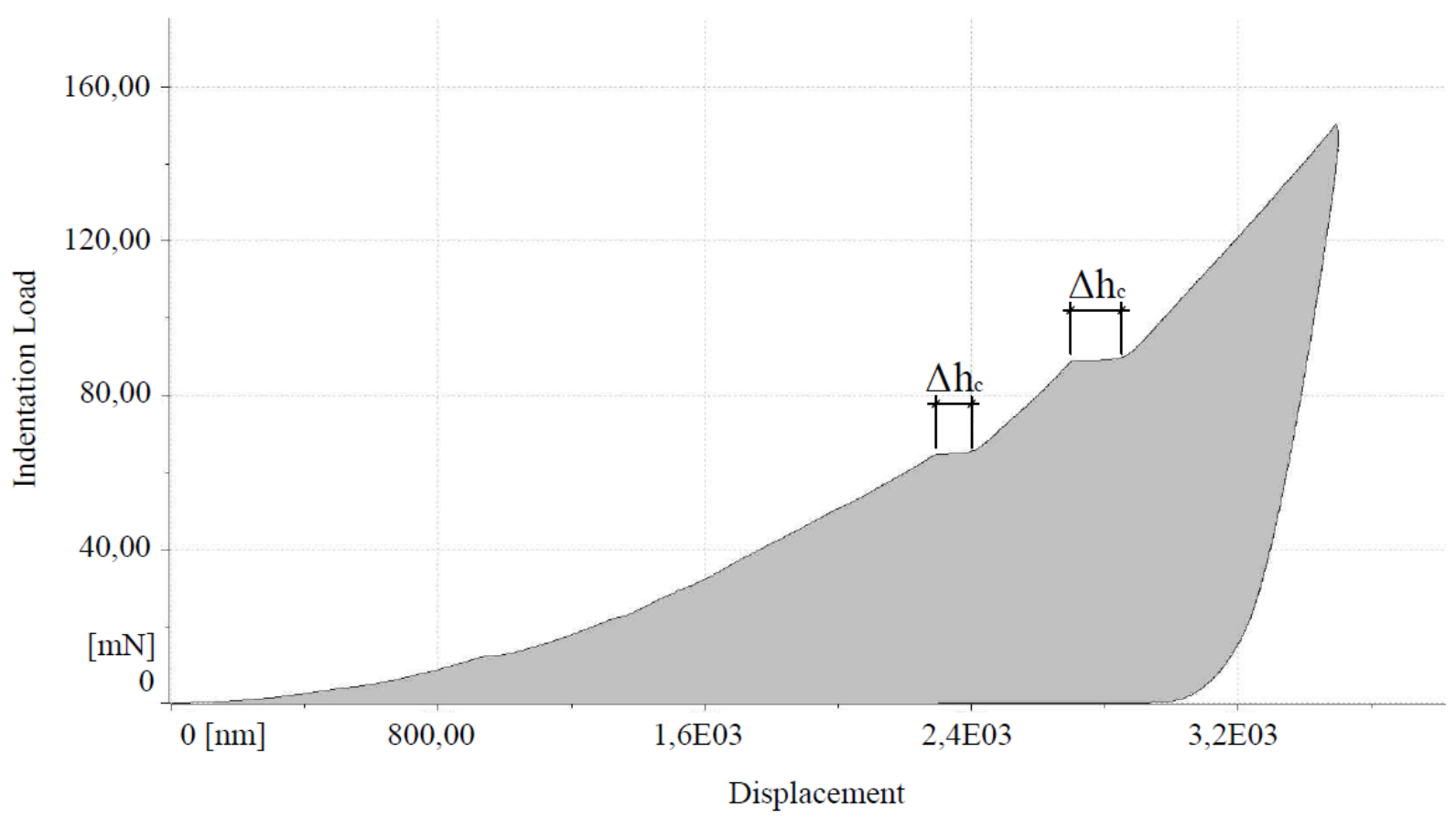

FIGURE 3. Indentation curve showing the initiation and propagation of cracks (pop-in effect). Initiation of a crack causes a sudden increase in contact depth $h_{\mathrm{c}}$ which affects contact area $A$, and consequently hardness $H_{\mathrm{IT}}$ and reduced modulus $E_{\mathrm{r}}$. During initiation of cracks the energy is dissipated and the total energy is increasing.

impossible to determine the fracture toughness by the VIF method. An alternative option is a method based on the dissipation of energy. The method uses the energy released during the crack initiation and growth, which is called the fracture energy $U_{\text {frac }}$.

The release of energy is obvious from the indentation curve in Fig. 3. The crack initiation causes a shift of the loading curve $\Delta h_{\mathrm{c}}$ (pop-in effect) and an increase in total energy of indentation, which is displayed as the area under the loading curve. The fracture energy can be separated from the total energy $W_{\text {tot }}[13]$ :

$$
W_{\mathrm{tot}}=W_{\mathrm{el}}+W_{\mathrm{pl}}+U_{\mathrm{frac}}+W_{\text {other }}
$$

where $W_{\mathrm{el}}$ is the energy of the elastic deformation, which is displayed as the area under the unloading curve, $W_{\mathrm{pl}}$ is the energy of the plastic deformation and $W_{\text {other }}$ are other energies, for example energy of creep or energy associated with changes in temperature. The sum of the fracture energy, the energy of the plastic deformation and other energies is called an irreversible energy. This is displayed as the area enclosed by the loading and unloading curve (grey area).

The energy of the plastic deformation cannot be derived directly from the indentation curve, so it is necessary to use a linear relationship between the ratio $W_{\mathrm{pl}} / W_{\text {tot }}$ and ratio $h_{\mathrm{f}} / h_{\max }[14]$ :

$$
\frac{W_{\mathrm{pl}}}{W_{\mathrm{tot}}}=(1+\lambda) \frac{h_{\mathrm{f}}}{h_{\max }}-\lambda
$$

where $h_{\mathrm{f}}$ is the final indentation depth, $h_{\max }$ is maximal indentation depth and $\lambda=0.27$. Other energies can be eliminated by test conditions or dwell time in the case of the creep.
If the fracture energy $U_{\text {frac }}$ is derived, the fracture toughness $K_{\mathrm{IC}}$ is calculated from formulas based on principles of linear elastic fracture mechanics (LEFM) [15]:

$$
\begin{aligned}
G_{\mathrm{C}} & =\frac{U_{\mathrm{frac}}}{A_{\mathrm{frac}}} \\
K_{\mathrm{IC}} & =\sqrt{E G_{\mathrm{c}}}
\end{aligned}
$$

where $A_{\text {frac }}$ is the area of the fracture whose initiation caused the energy dissipation. The method based on the dissipation of energy enables the determination of fracture toughness in the case of initiation of any cracks (Palmqvist, half-penny, median, delamination) in contrast to the VIF method.

It was found out that the values of the applied load for measuring the hardness and reduced modulus of a brittle material can significantly influence overall results of these characteristics, and consequently fracture toughness determined by the VIF method. The degree to which the fracture toughness is affected depends on the value of the applied load and a formula used for the calculation of $K_{\mathrm{IC}}$ because the exponent of the ratio $E / H_{\text {IT }}$ differs in formulas derived by different authors. In this test, the applied load (10$150 \mathrm{mN}$ ), was significantly lower than in experiments performed by other authors. The authors usually apply load 1-10 N which means that changes of hardness and reduced modulus will probably be more obvious.

The problem is even more augmented because authors do not use the same methods to determine the values of hardness and modulus. While some authors [8] use mean values of hardness and modulus 
cited in literature, others [2, 3] use the nanoindentation or they determine the parameters directly while testing the fracture toughness. Padmanabhan [10], for example, describes a hardening of enamel with increasing loading but the question is how much are the results influenced by values of hardness and modulus, which are determined in the same test as the fracture toughness. It is, therefore, necessary to provide these parameters by other means rather than directly from the test of the fracture toughness.

In this test, the choice of the loading velocity showed no significant influence on the outcome of the measurement. As in the previous experiment, changes can be more obvious if authors use the loading velocity greater than $450 \mathrm{mN} / \mathrm{min}$.

\section{Conclusions}

The present study assesses the brittle behaviour of enamel during nanoindentation tests and possibility of using the nanoindentation for a fracture toughness determination. The VIF method, almost solely used by other authors, was found to be inapplicable. Therefore, the alternative method based on a dissipation of energy was described. This method can eliminate some negative aspects of the VIF method. The factors influencing the overall results of the fracture toughness test were examined. As the values of the applied load were found to be the important factor of the fracture toughness determination by the VIF method, the choice of the loading velocity did not affect the result to any noticeable degree.

Further study will be dedicated to the determination of the stress intensity factor $K_{\mathrm{IC}}$ on the basis of the fracture energy $U_{\text {frac }}$ and fracture area $A_{\text {frac }}$. Since the fracture energy is relatively easy to determine, the measurement of the fracture area will be crucial.

\section{ACKNOWLEDGEMEnTS}

The financial support by Faculty of Civil Engineering, Czech Technical University in Prague (SGS project No. SGS17/168/OHK1/3T/11) is gratefully acknowledged.

\section{REFERENCES}

[1] D. J. Chiego. Essentials of Oral Histology and Embryology-E-Book: A Clinical Approach. Elsevier Health Sciences, 2014.

[2] S. Park, J. Quinn, E. Romberg, D. Arola. On the brittleness of enamel and selected dental materials. Dental materials 24(11):1477-1485, 2008. DOI:10.1016/j.dental.2008.03.007.

[3] Q. Zheng, H. Xu, F. Song, et al. Spatial distribution of the human enamel fracture toughness with aging. Journal of the mechanical behavior of biomedical materials 26:148-154, 2013. DOI:10.1016/j.jmbbm.2013.04.025

[4] S. Hayashi-Sakai, J. Sakai, M. Sakamoto, H. Endo. Determination of fracture toughness of human permanent and primary enamel using an indentation microfracture method. Journal of Materials Science: Materials in Medicine 23(9):2047-2054, 2012. DOI:10.1007/s10856-012-4678-3

[5] H. Xu, D. Smith, S. Jahanmir, et al. Indentation damage and mechanical properties of human enamel and dentin. Journal of Dental Research 77(3):472-480, 1998. DOI:10.1177/00220345980770030601.

[6] F. Sergejev, M. Antonov. Comparative study on indentation fracture toughness measurements of cemented carbides. Proc Estonian Acad Sci Eng 12(4):388-398, 2006. DOI:10.1111/j.1551-2916.2006.01482.x.

[7] G. D. Quinn, R. C. Bradt. On the vickers indentation fracture toughness test. Journal of the American Ceramic Society 90(3):673-680, 2007. DOI:10.1111/j.1551-2916.2006.01482.x

[8] R. Hassan, A. Caputo, R. Bunshah. Fracture toughness of human enamel. Journal of Dental Research 60(4):820 827, 1981. DOI:10.1177/00220345810600040901.

[9] W. C. Oliver, G. M. Pharr. Measurement of hardness and elastic modulus by instrumented indentation: Advances in understanding and refinements to methodology. Journal of materials research 19(1):3-20, 2004. DOI:10.1557/jmr.2004.19.1.3

[10] S. K. Padmanabhan, A. Balakrishnan, M.-C. Chu, et al. Micro-indentation fracture behavior of human enamel. Dental materials 26(1):100-104, 2010. DOI:10.1016/j.dental.2009.07.015.

[11] P. Hájková, A. Jíra. Micromechanical analysis of complex structures by nanoindentation. In Key Engineering Materials, vol. 731, pp. 60-65. Trans Tech Publ, 2017. DOI:10.4028/www.scientific.net/KEM.731.60

[12] A. Jíra, J. Němeček. Nanoindentation of human tooth dentin. In Key Engineering Materials, vol. 606, pp. 133-136. Trans Tech Publ, 2014. DOI:10.4028/www.scientific.net/KEM.606.133

[13] J. Chen, S. Bull. Indentation fracture and toughness assessment for thin optical coatings on glass. Journal of Physics D: Applied Physics 40(18):5401, 2007. DOI:10.1088/0022-3727/40/18/S01

[14] Y.-T. Cheng, Z. Li, C.-M. Cheng. Scaling relationships for indentation measurements. Philosophical Magazine A 82(10):1821-1829, 2002. DOI: $10.1080 / 01418610208235693$

[15] E. Rocha-Rangel. Fracture toughness determinations by means of indentation fracture. In Nanocomposites with unique properties and applications in medicine and industry. InTech, 2011. 